\title{
MÉTHODE D'ANALYSE DU NOMBRE DE CHROMOSOMES DANS LES CELLULES GERMINALES DU BELIER
}

\author{
PAR
}

\section{O. BOMSEL-HELMREICH}

Station de Recherches de Physiologie animale, Centre National de Recherches Zootechniques, Jouy-en-Josas.

I a constance du nombre chromosomique dans une même espèce est généralement admise, mais cette position très stricte paraît susceptible de révision à la suite de travaux récents. D'une part, des cas d'hétéroploïdie spontanée, ont été signalés; il s'agit généralement d'embryrons triploïdes, ou plus rarement d'aneuplö̈des (chez la Souris, BEATTy et FischBERG I949, I95I); d'autre part, de nombrenx auteurs ont décrit dans différents tissus une aneuploïdie naturelle : le nombre de chromosomes n'est pas le multiple exact du nombre haploïde. BEAT'rY (I954) dans sa revue générale sur ce sujet indique qu'apparemment le nombre de chromosomes varie assez peu dans l'embryon non différencié; cette variation semble commencer à se manifester entre la gastrulation et la formation des ébauches ; BEA'T'TYY dit très justement que, s'il est peut-être trop tôt pour conclure que la variation du nombre de chromosomes est une caractéristique de tous les tissus somatiques des Mammifères, du moins, tous les travaux récents s'orientent dans ce sens. Par contre, la stabilité du nombre diploïde dans le tissu germinal n'a jamais été mise en cause. C'est pour cette raison que nous avons choisi le testicule pour vérifier le nombre de chromosomes du Mouton.

Un certain nombre d'auteurs ont étudié les chromosomes du Mouton, l'ensemble de leurs résultats figure sur le tableau I.

On voit qu'il n'existe pas une identité de vue entre les différents auteurs, les nombres les plus fréquemment retenus étant 54 ou 60 .

BERRY explique ces divergences par une différence d'interprétation : les 6 chromosomes en forme de $\mathrm{V}$ seraient comptés pour I2 chromosomes en bâtonnet. Novikov et BRUCE voient 60 chromosomes en bâtonnets; ils interpréteraient donc les $6 \mathrm{~V}$ comme I2 bâtonnets se chevauchant par une extrémité ; cependant une telle explication n'est pas valable pour toutes les données antérieures; en effet, KR.ILIINGER compte 60 chromo- 


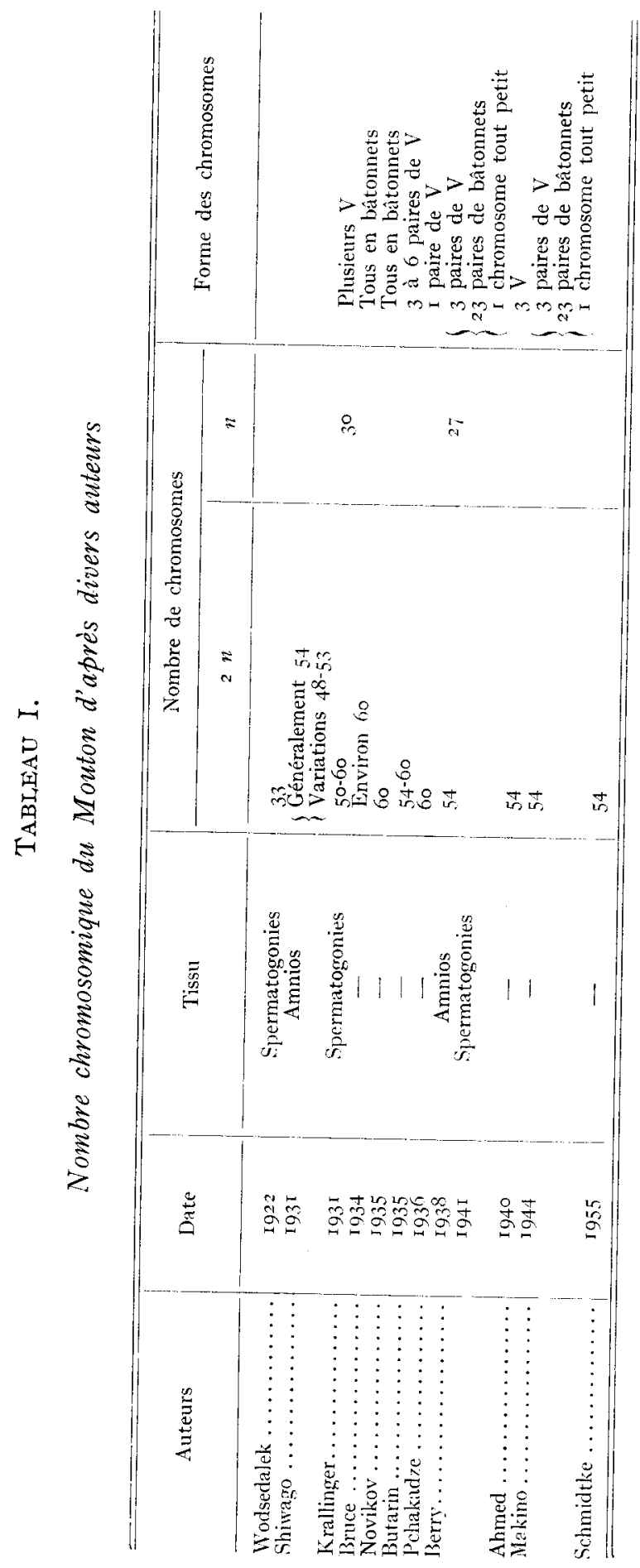


somes, tout en signalant plusieurs $V$; AHMED, au contraire, ne signale que $3 \mathrm{~V}$ et compte pourtant 54 chromosomes.

Il est frappant de constater, en étudiant les protocoles expérimentaux, que, le plus souvent, les auteurs n'indiquent pas à partir de combien de numérations ils ont déterminé le nombre diploïde caractéristique de l'espèce ; et quand le nombre est donné il s'avère qu'il n'a été fait qu'un petit nombre de comptages; BERRY seul indique avoir compté pour le Mouton "plus de 65 divisions spermatogoniales"; cet auteur, du reste, ne signale pas de variation. C'est pourquoi il nous a semblé nécessaire de baser nos observations sur un nombre important de numérations.

\section{I. - MATÉRIEL ET MÉTHODES}

\section{A. - Matériel.}

Nous avons utilisé au total I2 Béliers soit:

7 Béliers Ile-de-Iirance :

I âgé de II semaines,

2 âgés de 4 mois I $/ 2$,

I âgé de 6 mois,

2 âgés de 8 mois,

I âgé de 9 mois,

I Bélier L,imousin de 6 mois;

I Bélier Southdown de 8 mois ;

3 Béliers Mérinos d'Arles âgés de :

6 mois,

6 mois $\mathrm{I} / 2$;

II mois.

et compté 567 mitoses goniales.

Pour nous permettre de vérifier notre technique d'écrasement sur un matériel autre que le Bélier, nous avons également examiné 3 Rats Wistar âgés de 3 mois et compté I64 mitoses spermatogoniales sur ces animanx.

\section{B. - Les méthodes utilisées el leur valeur.}

\section{I. - Importance du délai de fixation après la mort.}

I a plupart des auteurs étudiant les chromosomes signalent combien une fixation rapide des tissus est importante pour 1'observation des mitoses. R. ORTAVANT (communication personnelle) a constaté des anomalies cytologiques dans les cellules germinales du Bélier quand la fixation est faite plus de 3 minutes après la mort de l'animal; des variations du nombre de chromosomes pourraient en résulter. 
Pour éclaircir ce point, nous avons comparé la fréquence des mitoses et la facilité de dénombrement des chromosomes à des temps variables après la mort. A cet effet des fragments de testicules des deux Béliers Mérinos d'Arles MA2 et MA3, ont été fixés, soit dans les trois minutes qui suivent l'abattage, soit une, deux, trois et huit heures après la mort, le testicule étant conservé tel quel dans l'intervalle à une température de $20^{\circ} \mathrm{C}$. Les prélèvements étaient fixés au Bouin-Hollande et la coloration faite par la méthode Feulgen-Bleu Alcyon ; les coupes servant aux numérations étaient d'une épaisseur de ro \%. Pour chaque temps nous avons compté les mitoses de 350 tubes séminifères, les tubes étant de section ronde et d'aspect normal et les coupes venant chaque fois de 2 régions différentes du testicule.

Nous avons compté le nombre de mitoses, prophases et métaphases spermatogoniales, méioses et stades du cycle spermatogénétique. Jusqu’à trois heures après la mort, il n'y a pas d'altération morphologique sensible des tissus, le nombre de mitoses ne diminue considérablement que 8 heures après la mort. I,e nombre de noyaux en pycnose reste sensiblenıent égal à celui du temps $O$ même à 8 heures.

\section{2. - Dénombrement des chromosomes.}

a) Difficultés propres aux Mammifères.

Chez tous les Mammifères, la détermination du nombre exact de chromosomes est difficile. Ce nombre est très grand, en général il dépasse la trentaine; aussi les mitoses goniales, telles qu'on les observe sur des coupes sériées, ne se prêtent guère à une détermination aisée des chromosomes. Il faut une véritable reconstruction dans l'espace de chaque noyau en prophase ou en métaphase; en outre chez las Ovins, les chromosomes ont tendance à se chevaucher en prophase comme en métaphase.

\section{b) La méthode classique et sa critique.}

Depuis une dizaine d'années, la plupart des cytologistes qui font des numérations de chromosomes chez les Mammifères utilisent la technique d'écrasement, en particulier tous les auteurs que nous citons depuis 195I. Par ailleurs, le tissu testiculaire des Mammifères étant difficile à écraser, depuis les travaux de Hsu et Pomerat (I953), on fait généralement précéder la fixation par un séjour dans une solution hypotonique qui facilite l'étalement en agissant sur le fuseau achromatique.

Les techniques d'écrasement que BeLIING employa en premier en 1926, sur du matériel végétal, donnent des résultats meilleurs que les déterminations à partir de coupes histologiques. I,es prélèvements de tissus sont fixés dans 1'alcool acétique; ensuite ils sont écrasés directement 

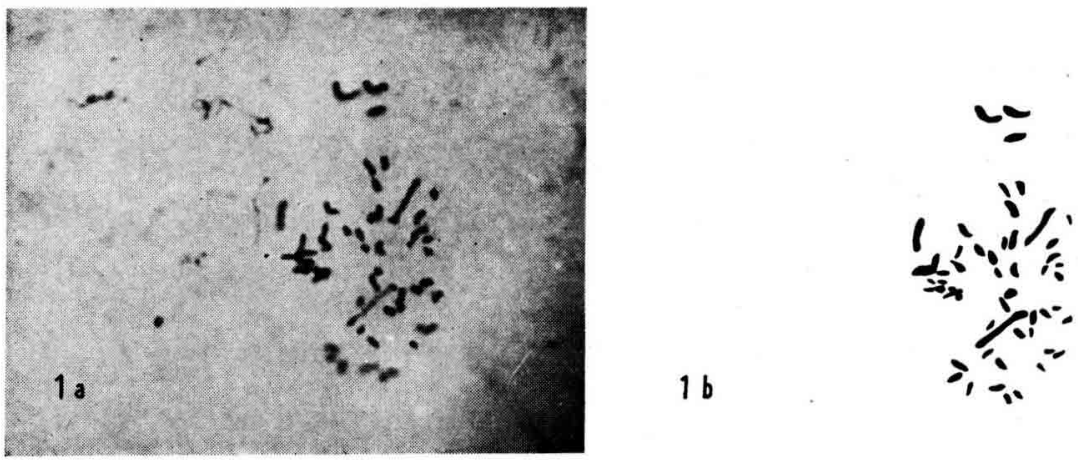

FIG. I. - Mitose spermatogoniale avec 52 chromosomes :

a) photographie. - b) interprétation graphique traduisant les divers plans de l'image microscopique.
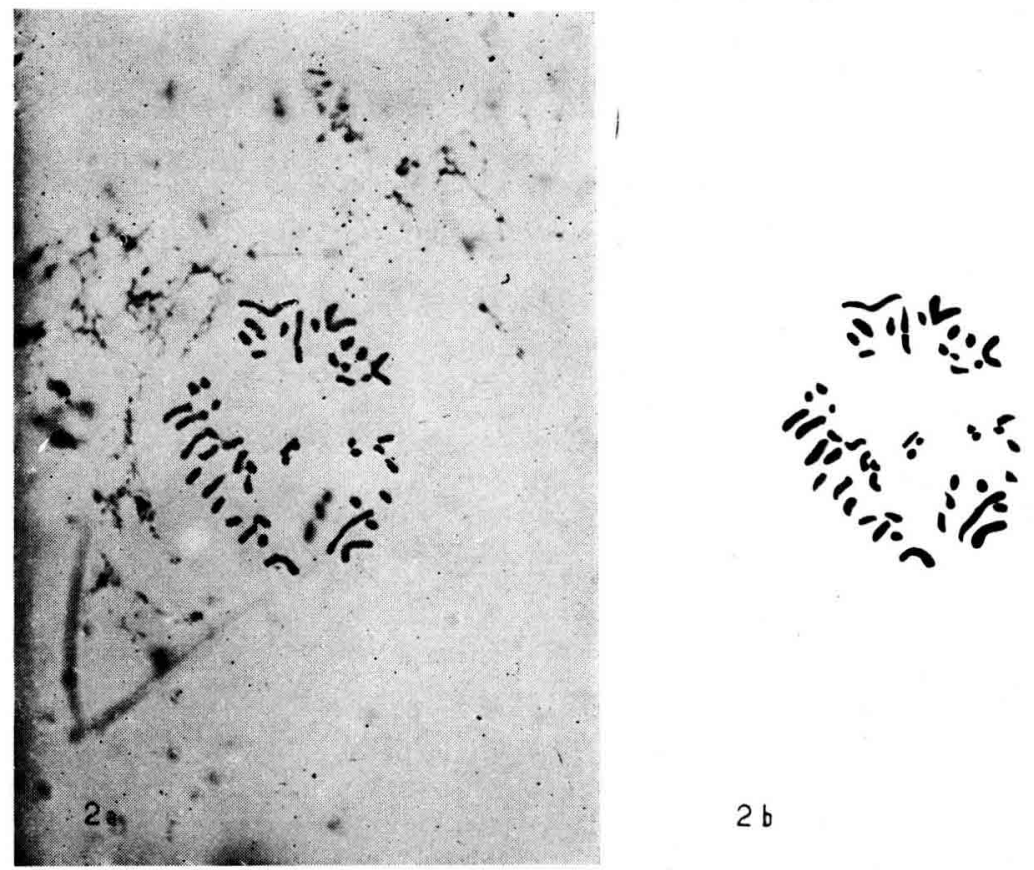

Fig. 2. -... Mitose spermatogoniale avec 52 chromosones:

-.- Les 6 chromosomes en $V$ sont particulièrement nets.

a) photographie. - b) interprétation graphique 
dans un colorant acétique, ou bien ils sont colorés d'après la méthode de Feulgen et écrasés ensuite; mais ces préparations pâlissent rapidement. I,es auteurs désirant des préparations permanentes, sont obligés de décoller lame et lamelle après l'écrasement pour monter la préparation dans le baume du Canada.

Le décollement de la lamelle et de la lame pour le montage dans le baume du Canada est une opération hasardeuse qui peut facilement produire des déchirements dans l'écrasement et par conséquent des pertes de chromosomes. Nous avons recherché un milieu liquide de conservation permettant un maintien de la coloration pendant plusieurs semaines sans déplacement de la lamelle après l'écrasement.

Nous avons utilisé la technique suivante:

Les tubes séminifères séjournent immédiatement après l'abattage ou la castration pendant 20 minutes dans une solution de chlorure de sodium hypotonique à $0,45 \mathrm{p}$. Ioo à $3^{\circ} \mathrm{C}$ (VFNGE). Ils sont ensuite fixés à 1'acool acétique 3 / I puis colorés in toto par la méthode de Feulgen avec un temps d'hydrolyse court ( 7 minutes à $5^{\circ}$ ); ils passent dans un bain d'acide lactique à $40 \mathrm{p}$. IOO ( 35 mintıtes) d'acide acétique à $45 \mathrm{p}$. IOO (I 5 minutes), de chloral-phénol d'Amann ( ${ }^{1}$ ) (I minute). Enfin ils sont écrasés avec la pointe d'une aiguille entre lame et lamelle dans une goutte de baume mêlée à une goutte de salicylate de méthyle. On lute la préparation au IDu Noyer et au vernis à ongles.

Ces préparations en milieu liquide se conservent plus d'un an sans décoloration.

c) Difficultés propres au Bélier et technique originale de préparation des objets.

Comme nous le verrons plus loin, dès le début de notre travail, nous avons observé une variation manifestement erronée du nombre de chromosomes ; un étalement insuffisant en était la raison, sans qu'il soit possible d'y remédier par une pression d'écrasement élevée. Aussi avons nous été amenés à améliorer notre technique de la façon suivante :

Nous avons cherché un facteur agissant sur la viscosité du cytoplasme et les liaisons intercellulaires. qui faciliterait ainsi les dissociations cellulaires au moment de l'écrasement. L'hyaluronidase en solution dans le bain hypotonique ( 500 unités VRU dans 5 cc de solution hypotoniqueà 0,45 p. roo de chlorure de sodium), s'avéra très efficace. En même temps nous avons oxygéné cette solution, pour établir une véritable survie des fragments testiculaires en présence d'hyaluronidase en milieu physiologique.

L'action conjuguée de l'hypotonie et de l'hyaluronidase s'est révélée très intéressante, mais seulement en milieu oxygéné. Non seulement la numération des chromosomes devenait plus aisée, car l'écartement

(1) Melange d'hydrate de chloral crist. et de phétol crist. $2 / 1$. 


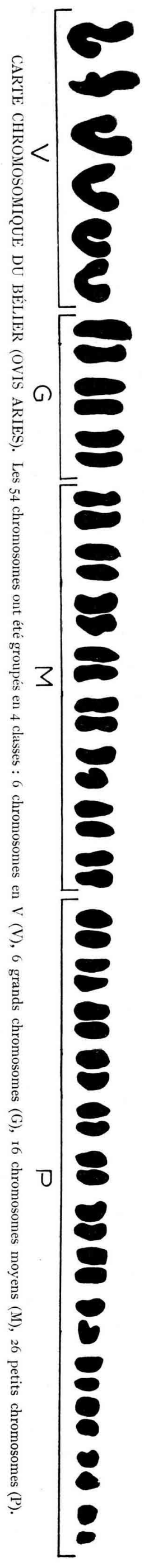




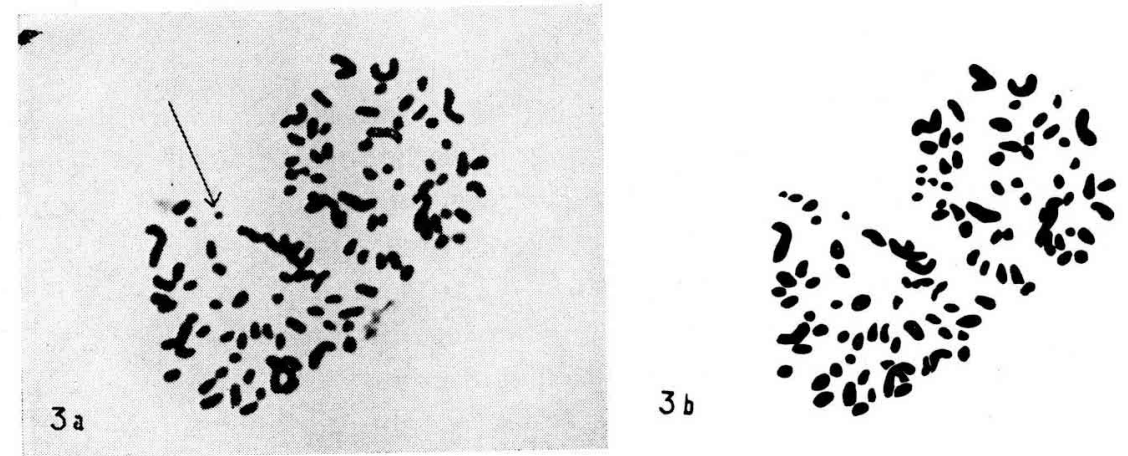

FIG. 3. - Deux mitoses spermatogoniales de 54 chromosomes côte à côte :

la fliche indique le plus petit chromosome; - a) photographie. - b) interprétation graphique.
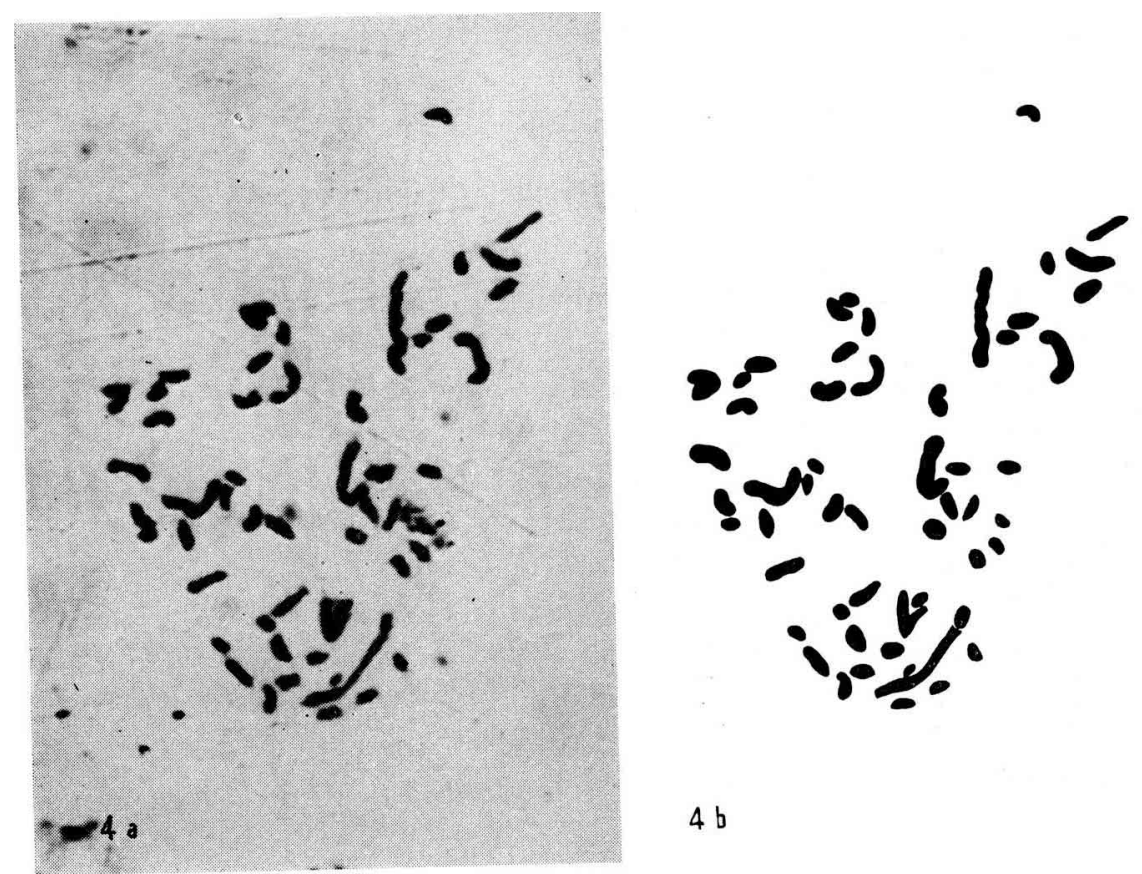

FIG. 4. -- Mitose spermatogoniale de 55 chromosones :

a) photographie. - - b) interprétation sraphique. 
entre chromosomes devenait plus grand, mais le nombre des mitoses utilisables augmentait. C'est une amélioration importante, car une grande sévérité dans le choix des mitoses prises en considération, a pour contrepartie une lenteur considérable dans l'accumulation des analyses, de 90 à 95 p. Ioo des images devant être éliminées.

d) Critères d'analyse.

I.es numérations de chromosomes ont été faites sur des prophases et des métaphases de mitoses spermatogoniales à tous les stades du cycle de la spermatogénèse ; c'est-à-dire sur des spermatogonies $\mathrm{A}$, intermédiaires ou B suivant la terminologie de R. ORTAVANT (I958).

Nos critères d'analyse étaient les suivants :

- Cellules en division nettement isolées les unes des autres, ne se trouvant pas sur le bord d'une plage d'écrasement.

- Critère "esthétique": ensemble des chromosomes de la mitose groupés sans rupture apparente dans un cercle dont le centre est celui de la plaque mitotique; pas de chromosomes isolés à proximité de la mitose considérée.

- Chromosomes nettement séparés les uns des autres.

-- Présence de 6 chromosone en $\mathrm{V}$.

- Contrôle de nos numérations par un deuxième observateur, notamment dans le cas des gonies à nombre aberrant.

\section{II. - RESULTATS}

\section{A. - La carte chromosomique.}

Afin de mieux connaître la morphologie des chromosomes du Bélier, nous avons établi à partir d'agrandissements photographiques une carte chromosomique.

Dans une spermatogonie normale de 54 chromosomes, il y a 6 chromosomes en $\mathrm{V}$ que l'on groupe facilement en trois paires homologues dont deux plus grandes et une plus petite. I,es autres chromosomes sont en forme de bâtonnets dont la taille décroît régulièrement; l'appariement deux par deux devient donc délicat. On peut cependant distinguer les 6 plus grands bâtonnets (les deux premiers sont nettement plus grands que les autres) et les 6 plus petits, presque punctiformes. Un dernier chromosome est nettement plus petit que tous les autres (fig. 2 et 3 ).

\section{B. - Résultats obtenus avec la méthode celassique d'écrasement. I. - Chez le Bélier.}

Une prenière série de 262 numérations faites sur 5 Béliers de race Ile-de-France ou l,imousins dont 1'âge allait de I I semaines à 8 mois mon- 

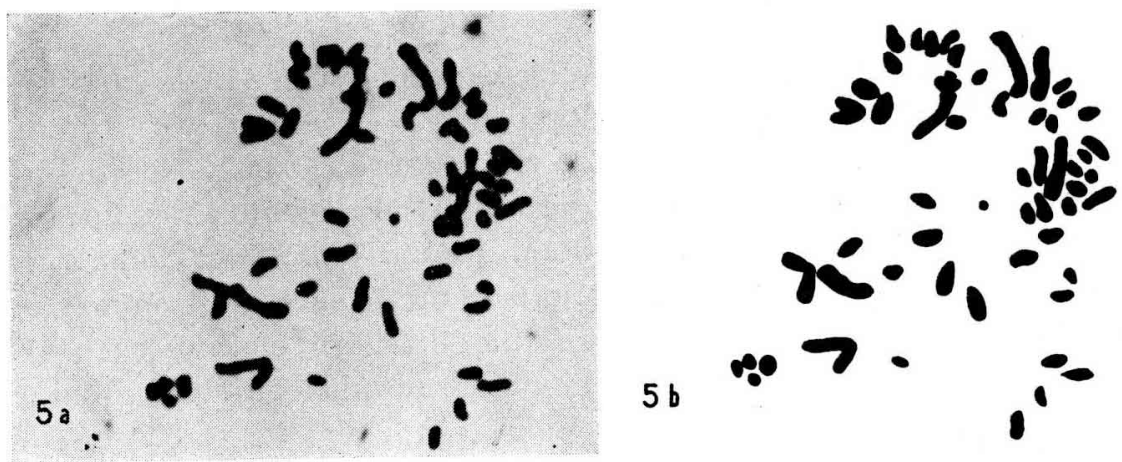

Fig. 5. - Mitose spermatogoniale de 56 chromosomes :

a) photographie. - b) interprétation graphique.
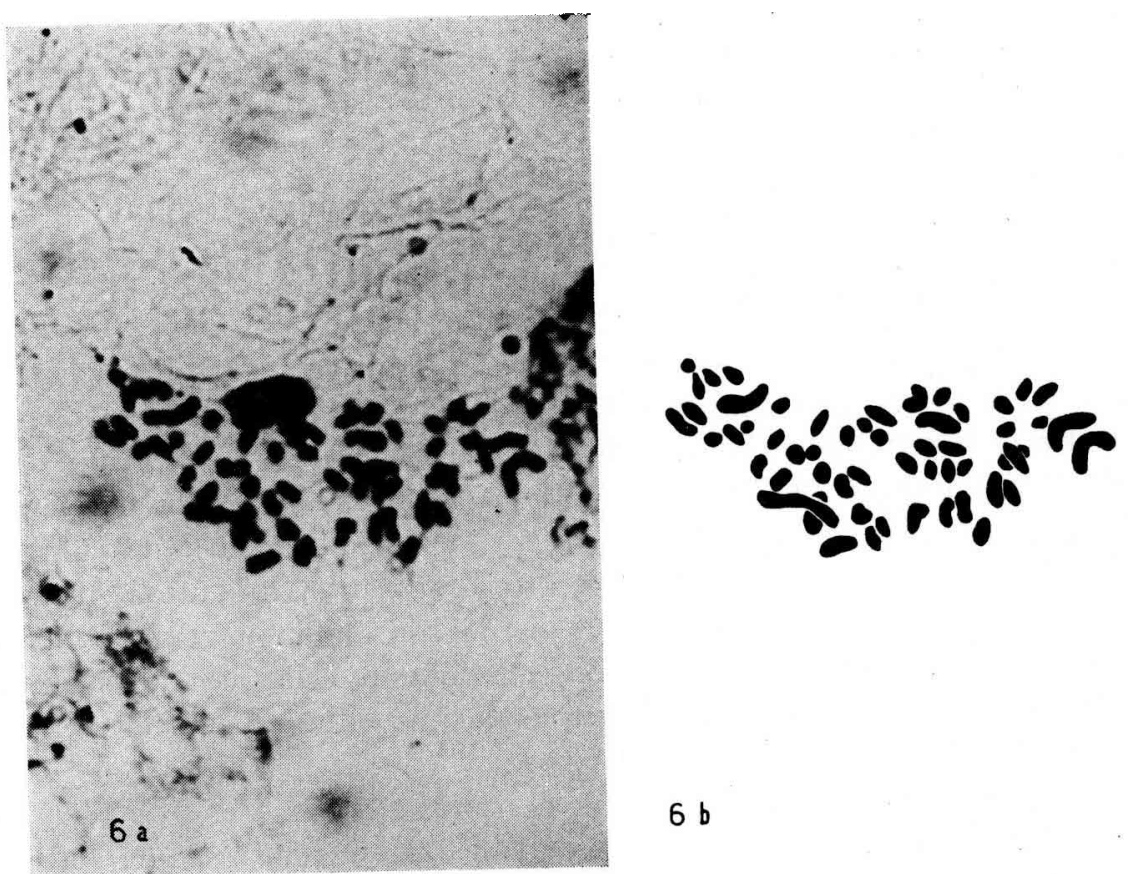

$6 b$

FIG. 6. - Mitose spermatogoniale de $5^{6}$ chromosomes :

a) photographie. - b) interprétation graphique. 
trait une variation importante du nombre diploïde. Le nombre de chromosomes variait de 45 à 62 avec un mode à peine marqué autour de 54 . I, origine de cette variation était-elle due uniquement à la technique?

\section{2. - Chez le Rat.}

Pour apprécier la valeur de cette variation, nous l'avons vérifiée sur un autre matériel biologique, le Rat.

Un nombre plus important de travaux a été fait sur le nombre de chromosomes du Rat (Rattus norvegicus) que sur ceux du Bélier. Ce nombre est fixé à 42, depuis les travaux de Minouchi (I928) qui donna à cette occasion une belle critique des résultats précédents. De nombreux auteurs ont repris le même sujet; en dernier lieu. TJ ro et LEVAN (1956) ont analysé à l'aide de plusieurs idiogrammes la morphologie des chromosomes du Rat. Le nombre indiqué est toujours nettement de 42 chromosomes.

Notre courbe de variation pour le Rat (nous avons utilisé an total I64 numérations) indique clairement qu'il n'y a pas de variation véritable du nombre de chromosomes; le mode de 42 est très net. Il y a une différence évidente entre cette courbe et les courbes des Béliers (fig. I3).

I a méthode classique est donc satisfaisante quand il s'agit du testicule du Rat; elle ne l'est pas pour le testicule du Bélier. Remarquons que les mitoses du Rat sont plus lisibles, car le tissu testiculaire se prête mieux à l'écrasement et l'étalcment des chromosomes est mailleur.

\section{C. - Résultats obtenus avec la terhnique originale de préparation.}

En utilisant l'action de l'hypotonie et de l'hyaluronidase en milieu oxygéné, l'étalement des chromosomes des mitoses goniales du Bélier atteignait une qualité égale, sinon supérieure, à celle obtenue chez le Rat par la traitement hypotonique seul. Nous avons alors étudié avec cette technique 7 Béliers de race Ile-de-France, Southdown et Mérinos d'Arles dont l'âge allait de 4 mois I/2 à II mois (Tableau II).

Nous avons compté environ 50 mitoses par animal. 'Toutes les courbes de fréquence des chromosomes ont une allure semblable et accusent un mode pour la valeur 54. Flles diffèrent donc de celles obtenues avec la méthode classique ; cependant la dispersion reste d'une dizaine de chromosomes (fig. 8-12).

Soulignons quelques aspects de ces courbes.

La technique de l'écrasement détruit évidemment tout repère topographique dans la préparation. I,es spermatogonies se trouvent, de par leur position dans le tube séminifère, proches les unes des autres et entrent souvent en division d'une manière synchrone. Aussi chaque fois que nous 
(IV, I959) ANALYSE DU NOMBRE DE CHROMOSOMES

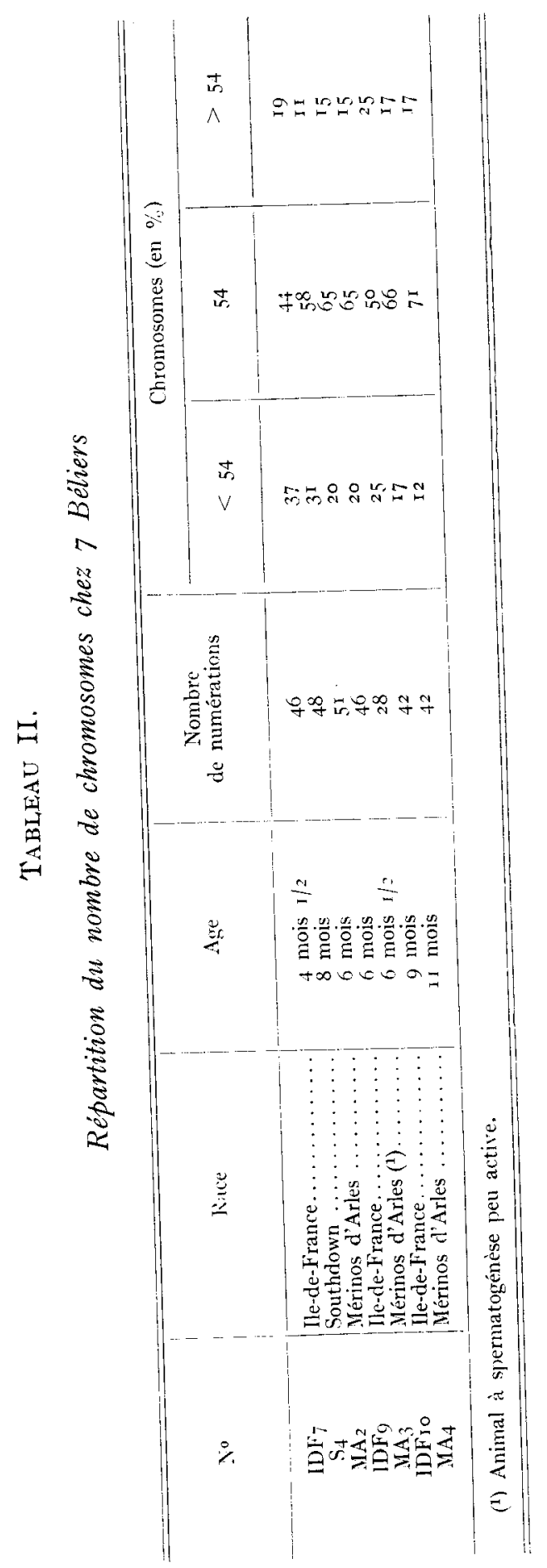



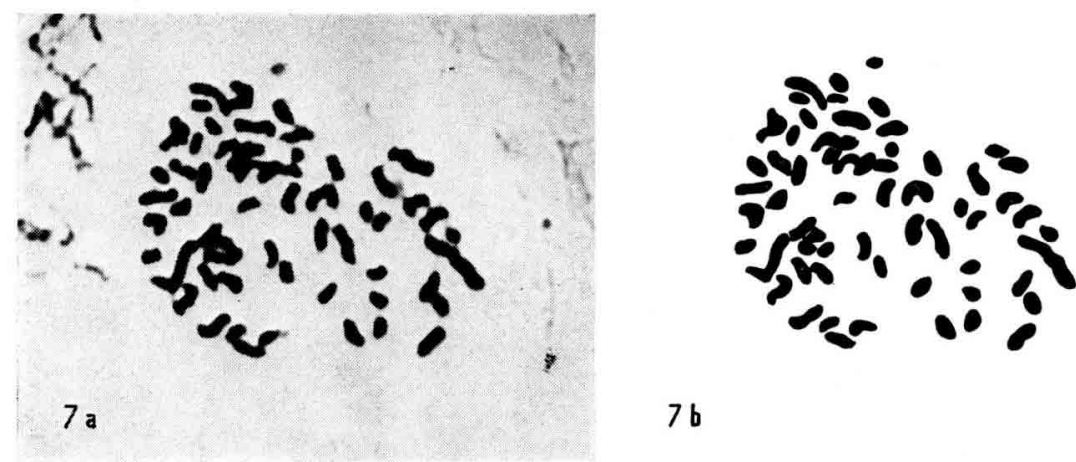

lici 7. - Mitose spermatogoniale de 60 chromosones :

a) photographie. - b) interpretation graphique.

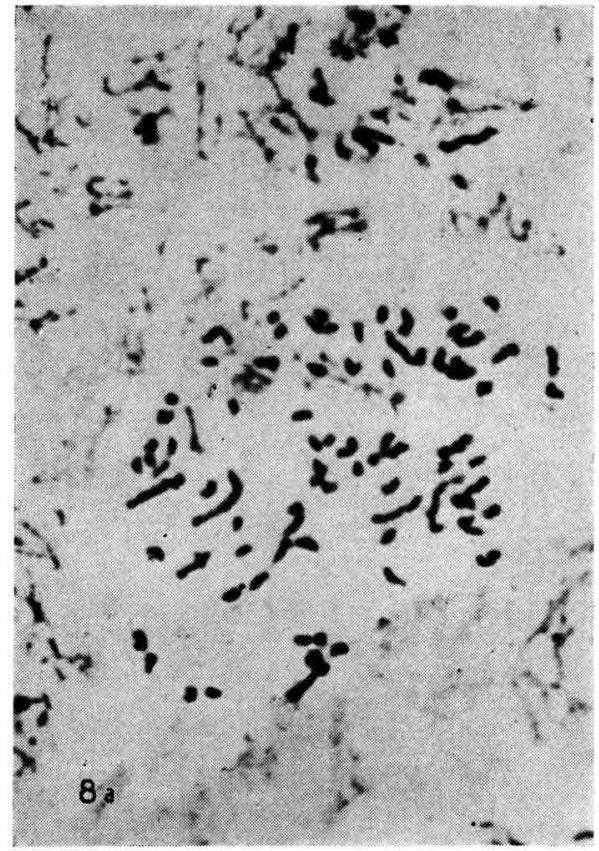

$7 b$$$
\text { 政 }
$$ 
avons trouvé une plaque mitotique de 108 chronnosomes, nous l'avons considérée comme étant composée de 2 mitoses voisines et non comme une gonie tétraploïde. Il en était de même pour les gonies, assez rares, où le nombre de chromosomes se place entre 78 et 108 chromosomes; nous ne les avons pas mentionnées sur nos courbes, les considérant comme une gonie normale et un fragment d'une gonie voisine.

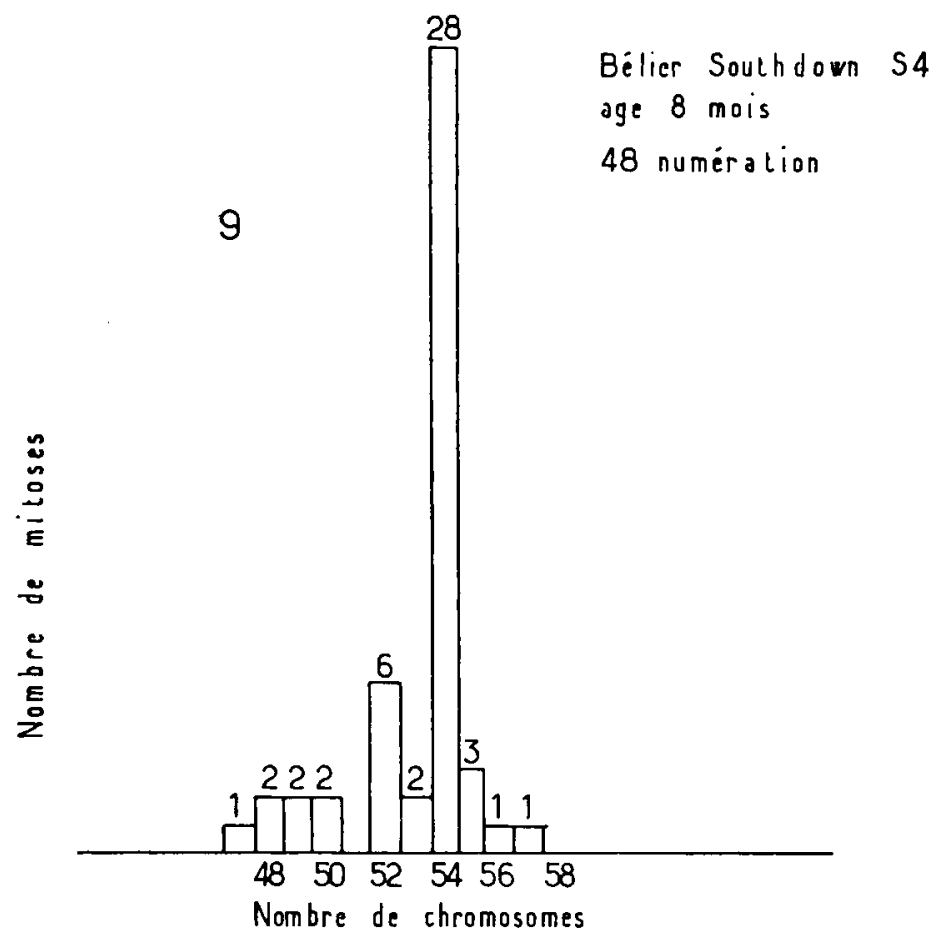

Fig. 9. - Distribution du nombre de chromosomes chez un Bélier Southdown ágé do 8 mois - . to numérations.

Nous avons trouvé cependant une dizaine de cas de gonies à 8 I chromosomes avec 9 chromosomes en $\mathrm{V}$; nous les signalons sans pouvoir affirmer leur caractère triploïde (fig. 8).

\section{1). - Étude des mitoses post-mortem dans le testicule de Bélier.}

Cependant notre technique originale d'écrasement rendait impossible une fixation rapide des tubes séminifères. Il se pouvait que l'altération des tissus après la mort était très rapide bien que nous n'ayons pas observé de variations du nombre de mitoses (nous l'avons vu précédemment). Le délai nécessaire à la préfixation entraînait-il des anomalies cytologiques importantes? 
Aussi parallèlement à l'étude de la frécuence des mitoses post-mortem, nous avons également fait des écrasements (50 à chaque temps) de fragments de tubes séminifères $\mathrm{I}, 2,3$ et 8 heures après la mort. Nous les avons comparés avec deux séries d'écrasements faits avec les deux techniques signalées jlus haut, l'une avec simple préfixation dans une solution

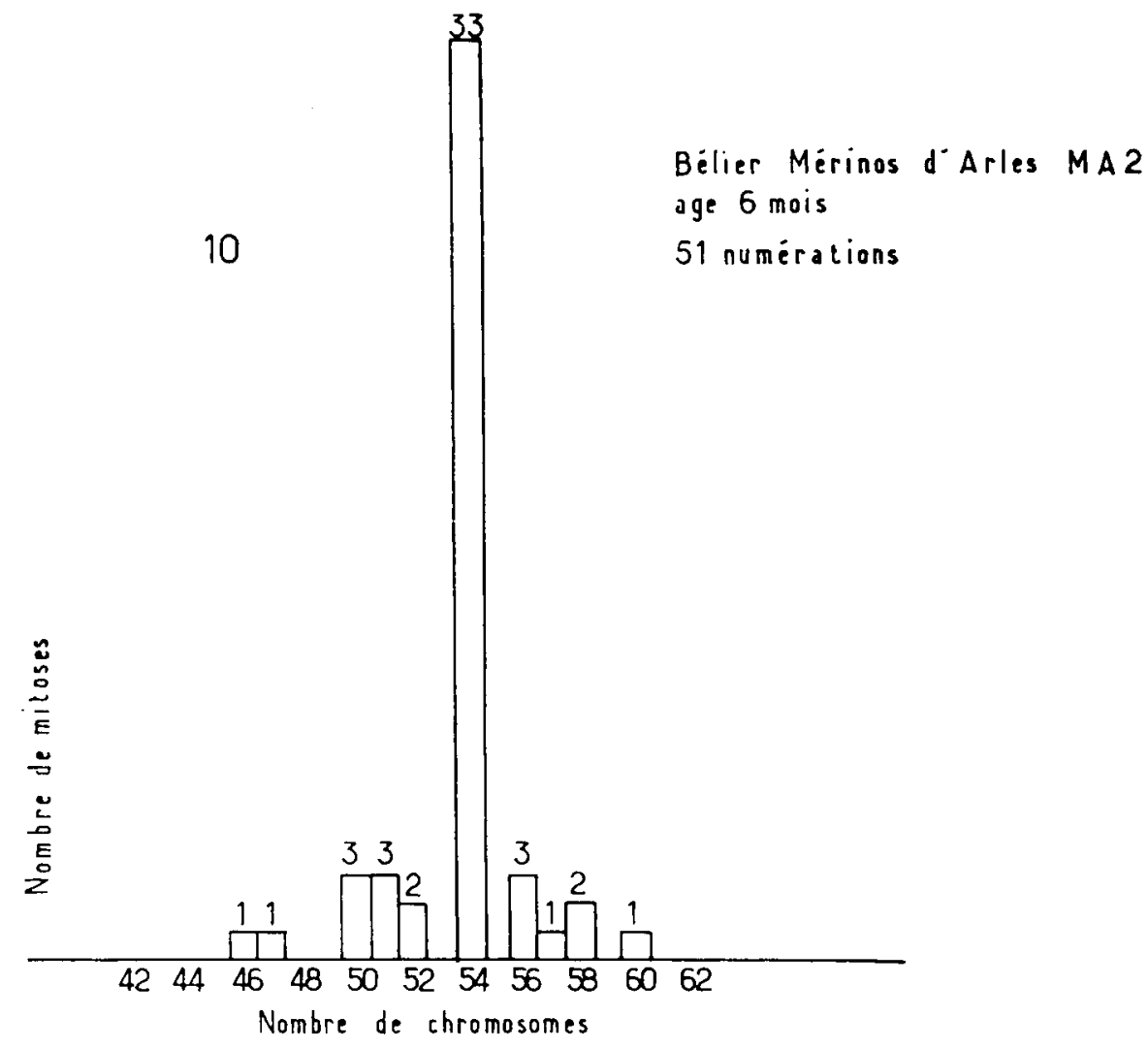

Firi. Io. - Distribution du nombre ale chromosones chez un Bélier Mérinos d'Arles igré de 6 mois - 51 nunciations.

hypotonique, l'autre avec préfixation dans une solution hypotonique avec oxygénation et hyaluronidase.

I.es écrasements confirmaient les indications fournies par les coupes : le nombre de mitoses spermatogoniales pour $\mathrm{I} h, 2 \mathrm{~h}$ et $3 \mathrm{~h}$ était comparable à celui du temps $\mathrm{O}$, (c'est-à-dire fixation à l'abattage). Pour I h et $2 \mathrm{~h}$, less mitoses spermatogoniales permettaient des numérations de chromosomes, mais à mesure que le temps de la fixation s'éloigne du moment de l'abattage, les chromosomes deviennent plus flous et se collent ks uns 
aux autres, rendant la lecture du nombre de chromosomes de plus en plus difficile; à partir de 3 heures, il n'est plus possible de faire des numérations, même en maintenant les fragments de testicule en milieu physiologique oxygéné.

\section{III. - DISCUSSION}

I,es variations inhabituelles du nombre de chromosomes que nous avons observées nous ont surpris. Plusieurs explications peuvent en être données.

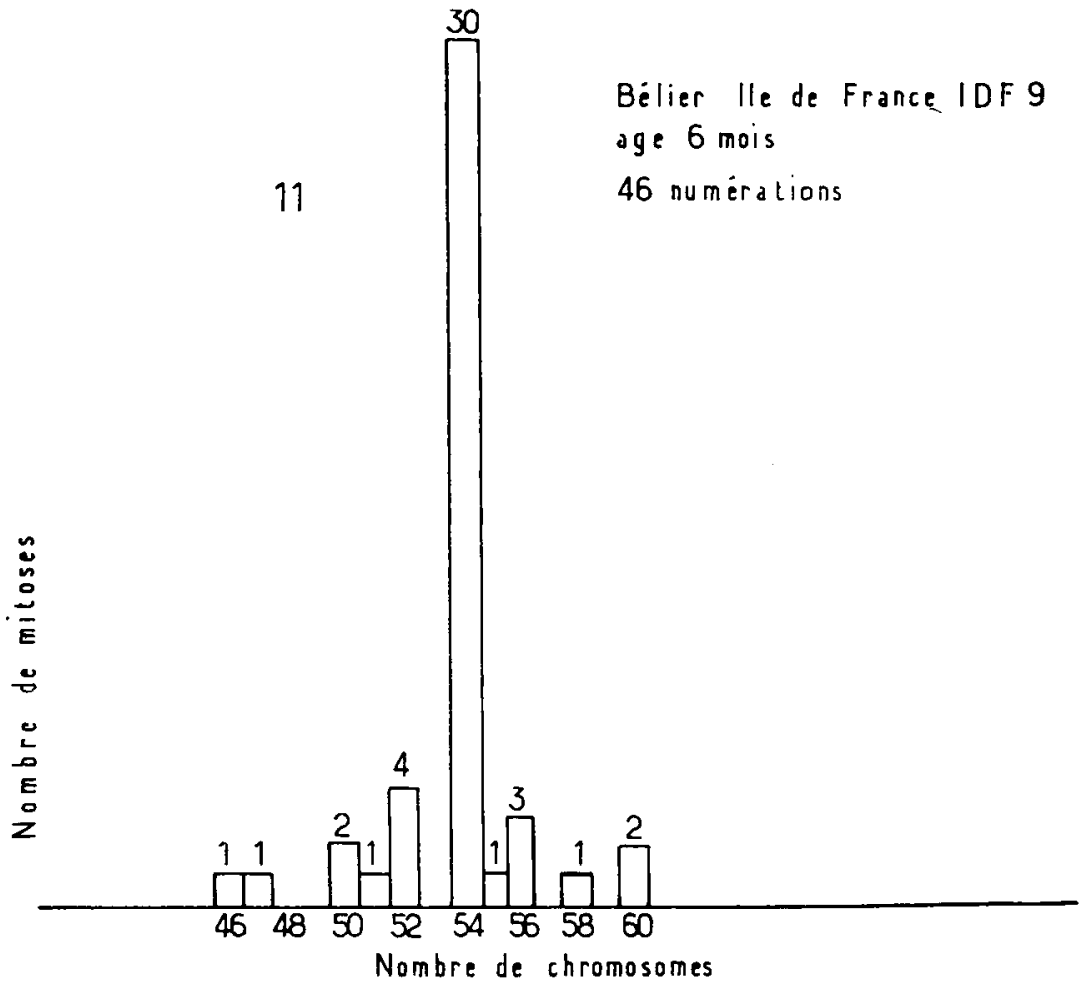

Fu. Ir. - Distribution du nombre de chromosomes che\% un Jiélier Ile-de-Jirance áge de 6 mois - 4h numérattions.

On est frappé tout d'abord par le fait qu'en modifiant la technique, on est arrivé à améliorer considérablement les analyses dans le sens d'une plus grande stabilité autour du nombre 54. On serait donc en droit de penser, que les variations encore observées tiennent à une technique défectueuse, bien qu'il ne nous semble pas possible de l'améliorer, compte tenu des multiples essais effectués.

Pour vérifier indirectement cette hypothèse, nous avons établi les cartes de chromosomes. I.es fractionnements ou agglutinations portaient 


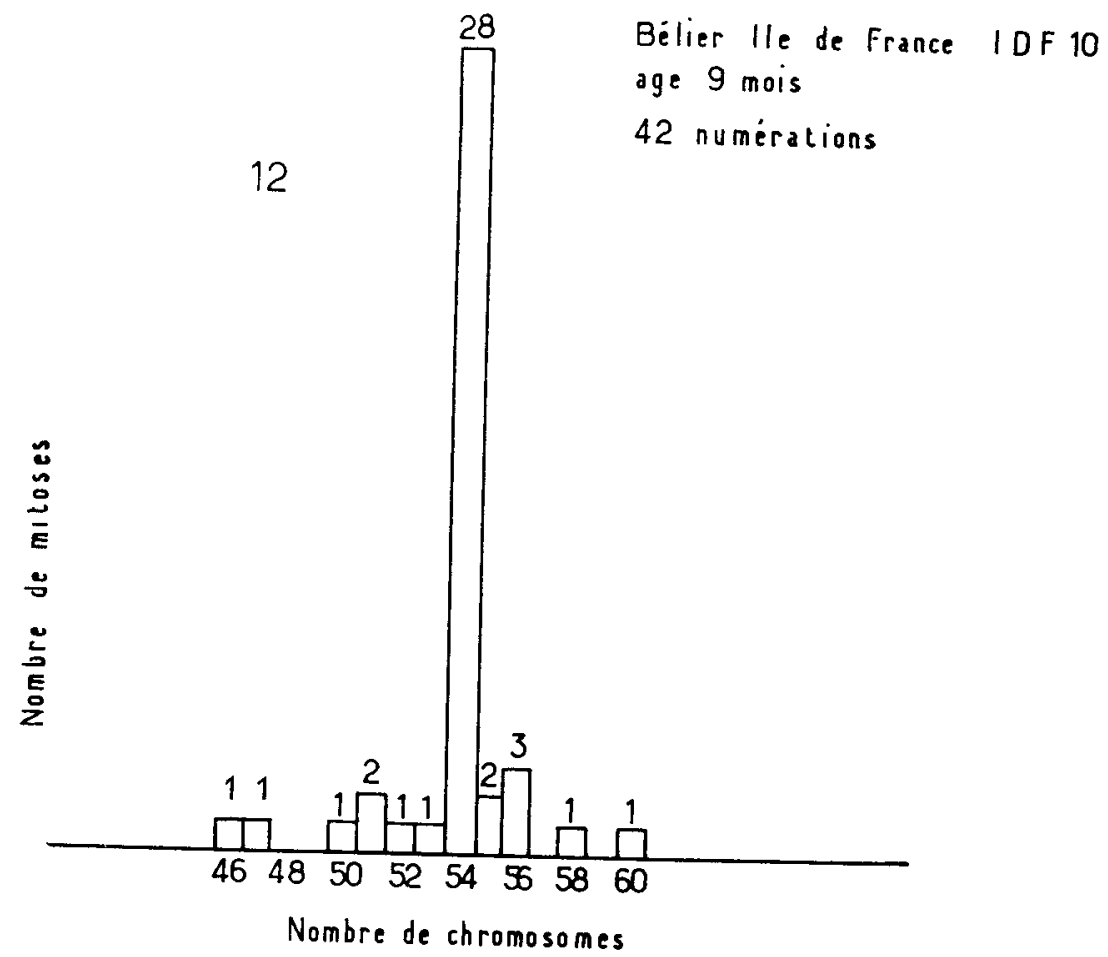

Fig. T2. - Distribution du nombre de chromosomes chez un Bélier Ile-de-France âgé de 9 mois - 42 numérations.

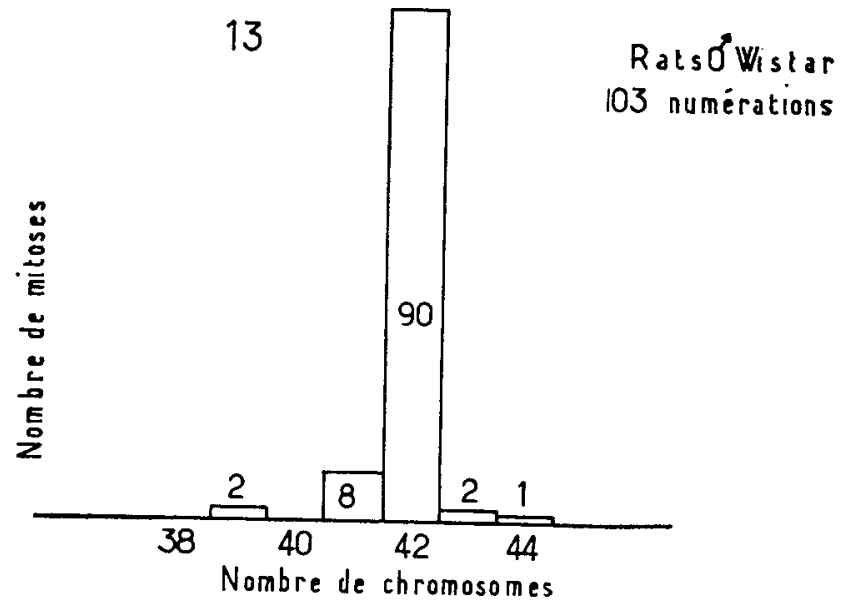

Fig. 13. - Distribution du nombre de chromosomes chez un Rat Wistar âgé de moins de 3 mois -103 numérations. 
uniquement sur les chromosomes moyens : il ne pouvait done s'agir d'un simple morcellement des grands chromosomes sous l'effet de l'écrasement.

Malheureusement une comparaison de la longueur totale des chromosomes est rendue aléatoire en raison des faibles différences de taille des chromosomes entre eux et de leur petite taille en général.

Des facteurs spécifiques pouvaient intervenir ici. En effet RoosisRUNGE (I953) avait montré que chez le Rat des divisions spermatogoniales avaient lieu plusieurs heures après la mort. Peut-être n'en était-il pas ainsi chez les Ovins? Une nécrose accompagnée d'une agglutination possible des chromosomes post-mortem intervenait peut-être rapidement? Nous avons vu que les modifications visibles ne se produisaient qu'après un délai nettement supérieur à celui nécessité par le traitement hypotoniehyaluronidase. Cependant, plus le délai après la mort est grand, plus les chromosomes ont tendance à s'agglutiner et l'absence d'oxygénation accuse cette tendance.

Des traitements pendant un temps très limité et le maintien du tissu dans un milieu oxygéné pendant la préfixation sont donc très importants.

Des modifications post-mortem rendraient compte du nombre inférieur à 54 mais n'expliqueraient pas les nombres supérieurs. Notons que la fréquence des figures à nombre supérieur à 54 n'augmente pas arec le délai post-mortem.

Il n'en reste pas moins vrai que les courbes de fréquence du nombre de chromosomes des mitoses spermatogoniales chez le Bélier, diffèrent sensiblement de celles du Rat.

Nous avons également pensé que les variations pouvaient être le reflet d'hybridation entre des races de Mouton à nombre différent de chromosomes. L,es premiers animaux étudiés provenaient en effet de races ovines résultant de croisements récents comme l'Ile-de-Iirance. Mais depuis nous avons utilisé non seulement des Béliers Ile-de-France mais aussi des Béliers de races fixées comme le Mérinos d'Arles ou très fixées comme le Southdown. N'ayant pas constaté de différence, nous pensons que l'origine de la variation du nombre ne se trouve pas dans des différences raciales.

I, 'âge et la maturité sexuelle ne sont pas non plus le facteur déterminant, contrairement à l'hypothèse de Schmidtke. Celui-ci explique les éventuelles variations du nombre de chromosomes trouvés par VENGE (I952) sur le I apin impubère et par lui-même (I955) sur le jetne Lapin et le jeune Bélier par une variabilité assez grande du tissu germinal avant la maturité sexuelle et qui disparaitrait ensuite. Nous avons nous-même examiné des Béliers impubères, mais nous avons abandonné ce matériel car nous ne pouvions être sûr de distinguer mitoses germinales et mitoses somatiques. 
E11 effet Clekmont et Perey (I957) ont prouvé chez le Rat impubère la présence dans un même tube séminifère de divisions de gonocytes et de divisions de cellules de soutien (futures cellules de Sertoli) qui sont des cellules somatiques.

Il n'y a pas non plus de relations entre cette variation et l'activité spermatogénétique saisonnière. Les abattages ont été faits tout le long de l'année, en photopériode courte comme en photopériode longue.

Enfin les numérations à nombre aberrant se trouvent indifféremment à tous les stades du cycle de la spermatogénèse, c'est-à-dire qu'il s'agit aussi bien des mitoses de gonies $A$ que de gonies $B$.

La variation est-elle due à la présence d'hétérochromosomes multiples? BERRY (I94I) et MAKINo (I95I) supposent que le chromosome X serait un chromosome rond nettement plus petit que les autres. Nous aussi avons presque toujours observé un tel chromosome; cependant, suivant le stade de la mitose et la qualité de l'écrasement, il arrive que l'on ne puisse pas le distinguer des chromosomes de la plus petite classe de taille; c'est pour cela "ule nous n'en avons pas fait un critère d'analyse. Par contre, nous avons observé deux ou trois cas où il y avait deux chromosones nettement plus petits que les autres.

Nous retrouvons dans les bivalents de la division des cytes I, à côté des trois bivalents nettement plus grands queles autres et quicorrespondent a ux trois paires de chromosomes en $\mathrm{V}$, un bivalent en forme de crochet tout à fait typique. Il provient sans doute de l'accouplement du plus petit chromosome avec un autre de taille moyenne, et pourrait correspondre aux hétérochromosomes $X Y$; nous avons trouvé parfois des plaques de bivalents comportant deux de ces crochets. I,e reste des bivalents est de taille régulièrement décroissante; de toute façon, il n'y a pas de 1lunération rigoureuse possible pendant la diacinèse en raison de leur contraction. Cette contraction plus ou moins grande rend toute individualisation et comparaison des bivalents incertaines.

Donc pour les raisons que nous venons d'énoncer, il ne semble pas que la présence d'hétérochromosomes multiples puisse fournir une explication aux variations observées.

Les variations seraient-elles dues à des transformations robertsoniennes? WAHRMANN et ZAHAVI (I955) observent un polymorphisme chromosomique des autosomes dans une population d'un petit rongeur Gerbillus Pyramidum. Ford, Hammerton et Sharman (I956-57) observent un polymorphisme semblable sur plusieurs populations d'un autre rongeur Sorex Araneus. Ce polymorphisme varie d'une année à l'autre. Cependant les variations des autosomes dans ces deux cas suivent les lois de ROBERTSON : le nombre de bras chromosomiques reste toujours le même.

Il n'en est pas ainsi dans nes variations, puisque l'un de nos critères, 
arbitraire et limité, il est vrai, est justement la présence constante de 6 chromosomes en V, c'est-à-dire que les variations jouent seulement sur los chromosomes en bâtonnets.

Ces deux cas de polymorphisme chromosomique chez les Mammifères sont à l'échelle des populations; le troisième cas cité chez les Mammifères est celui de l'homme par KoDANi (I957). Cet auteur détermine le nombre de chromosomes de $2 \mathrm{I}$ individus adultes : seize ont 48 chromosomes, quatre en ont 46 et un 47 . Cette différence du nombre proviendrait de deux petits autosomes surnuméraires qui sont tantôt présents, tantôt absents. I'auteur a déterminé ces nombres surtout à partir de bivalents des spermatocytes I, et nous avons vu qu'il est difficile de se prononcer dans les diacinèses, mais ces données sont confirmées par quelques numérations faites sur des spermatogonies.

Chez les Invertébrés, la récente publication de BERGFRARI) (I958) indique un polymorphisme à l'échelle individuelle. In effet, sur une population de Nemobius Sylvestris (Grylloidea), cet auteur trouve sur les mêmes larves, des mitoses spermatogoniales à nombre différent, le nombre de $V$ et de grands bâtonnets restant cependant toujours le même, un $V$ pouvant remplacer un bâtonnet.

Peut-être le phénomène que nous avons observé est-il d'ordre comparable.

\section{RÉSUMÉ,}

Les chromosomes de $5^{67}$ mitoses spermatogoniales provenant de douze Béliers de races différentes ont été comptés. Les numérations ont été faites sur des écrasements selon les techniques classiques après un séjour dans une solution hypotonique. La technique usuelle a été améliorée par l'emploi de l'hyaluronidase et d'une oxygénation pendant la préfixation.

Les numérations montrent que la classe la plus nombreuse est celle de 54 chromosomes; toutefois une variation allant de 46 à 60 chromosomes a été constatée (environ 17 p. Ioo des numérations ont moins de 54 chromosomes, $65 \mathrm{p}$. roo en ont 54 et I8 p. roo plus de 54 chromosomes). Des numérations faites chez le Rat n'ont, par contre, montré aucune variation autour de 42 chromosomes.

I.es causes des variations enregistrées sont discutées.

\section{SUMMARY}

Chromosome counts have been carried out on twelve rams of different breeds ; 567 spermatogonial mitoses have been studied. Counting was done on preparations obtained by the classic squash method: small pieces of testis are left in a hypotonic solution, fixed in acetic alcohol, Feulgen-coloured and squashed ; this technique has been improved by using hyaluronidase and oxygenation during prefixation in the hypotonic solution.

Chromosome counts showed that the most frequent class is 54 chromo- 
somes, although there was a variation between 46 and 60 chromosomes (about I7 p. Ioo of the numerations showed less than 54 chromosomes, 65 p. Ioo showed 54 chromosomes, I 8 p. Ioo more than 54 chromosomes). However, in contrast, counts made on the rat did not vary from 42 chromosomes.

The causes of these variations are discussed.

\section{RËFERENCES BIBLIOGRAPHIQUES}

AhMeI (I. A.). - The structure and behaviour of the chromosomes of the sheep during mitosis and meiosis. Proc. Roy. Soc., Edinb., 60, 260-270, I940.

BEATTY (R. A.). - How many chromosomes in mammalian somatic cells? Int. Rev. Cytol., 3, I77-I97, I954.

BeAtTY (R. A.) and FischbeRG (M.). - Spontaneous and induced triploidy in pre-implantation mouse eggs. Nature, 163, 807-808, I949.

BEATtY (R. A.) and FischberG (M.). - Heteroploidy in mammals. I. Spontaneous heteroploidy in pre-implantation mouse eggs. J. Genet., 50, 345-359, I 95 I.

BEIIING (J.). - The iron-acetocarmine method of fixing and staining chromosomes. Biol. Bull., 50, I60-162, I 926.

Bergerari (J.). - Polymorphisme chromosomique dans une population de Nemobius sylvestris (Grylloidea). C. R. Acad. Sici., 246, 3528353 I, I958.

BERRY (R. O.). - Comparative studies on the chromosome numbers in sheep, goat and sheep $\times$ goat hybrids. $J$. Hered., 29, 343-350, I938.

BERRY (R. O.). - The chromosome complex of domestic sheep (Ovis aries). J. Hered. 32, 26I-267, I 144 .

BrUCE (H. A.). - The spermatogenetic history in sheep. Ph. I). Thesis, Univ. of Pittsburgh Library, I935.

ButARIN (N.S.). - The chromosome complex of the arhar and the fat-rumped sheep. Trudy Kirgiz. kompl. Eksp.,4, 77, I933-34, in Anim. Breed. Abstr., 4, 45,1935 .

Butarin (N. S.). - The chromosome complex of Arkhar (O. pollii karelini Serv.), Kurdiuchny ram (Ovis steatopyga) and their $\mathrm{F}_{1^{-}}$hybrid. Dokl. Akad. N. S.S.S.R, N. S., 4, $287-290$. I 935 .

Ci.ermont (Y.) and PeRey (B.). - Quantitative study of the cell population of the seminiferous tubules in immature rats. Amer. J. Anat., 100, $24 \mathrm{I}-260,1957$.

Ford (C. F.), HaMERTON (J. L.) and Sharmax (G. B.). - Chromosome polymorphism in the common shrew. Nature, 180, 392-393, I957.

Hsu ('T. C.). - Mammalian chromosomes in vitro. The karyotype of Man. I. Hered., 43, I67-I 72 , I952.

Kodani (M.). - Three diploid chromosome numbers of man. Proc. Nat. Acad. Sci., 43, 285-292, 1957.

KRALi,increr (H. F..) - Cytologische Studien in einigen Haussäugetieren. Wiss. Arch. f. Landzirsth. Abt. B. Arch. I. Tierernähr. Tierzucht., 5, I27-I87, I93I.

Makryo (S.). - The chromosome complexes in goat and sheep and their relationship. Chromosome studies in domestic mammals II. Cytologia, 13, 39-54, 1944 .

Makino (S.). - A contribution to the study of the chromosomes in some asiatic mammals. Cytologia, 16, 288-30I, I95I.

Mrxouchi (O.). -- Spermatogenesis of the albino rat. Jap. J. Zool., 1, 235$254, \mathrm{Ig} 28$. 
Novikov (I. I.). - - Chromosomes in the spermatogenesis of interspecific hybrids of the European Mouflon and the domestic sheep (Merino). Dokl. Akad. N. S.S.S.R., N. S., 4, 93-94, I935.

OR'TAVANT (R.). - Déroulement et durée du cycle spermatogénétique chez le Bélier. I ${ }^{\text {ere }}$ partie. Ann. Zoot., 8, I83-244, I959.

PCHAKADZE (G. M.). - A new data about the chromosome number in domestic sheep. Dokl. Akad. N. J.3.S.R.. N. S., 3, 333-334, I936.

RoOsen-RUNGE (E. G.). - Post-mortem mitotic activity of spermatogonia and spermatocytes in the albino rat. Exp. Cell. Res., 4, 52-59, I953.

Schmidtke, (C.). - Über die Zahlenkonstanz der Chromosomen bei Säugetieren. Berliner und Münchner Tierärzlliche Wochenschrift, 23, 4II-422, 1955 .

Schmintke (C.). - Über die Anwendung von hypotonischen Lösungen bei der Darstellung von Säugetier-Chromosomen. Zeitschtt Tierziichtung Zïchtungsbiol., 64, 38I-388, I955.

Sharman (G. B.). - Chromosomes of the common shrew. Nature, 17\%, 94I942, I 956.

Shrwago (P. I.). - Karyotypische Studien an Ungulaten. I. Über die Chromosomenkomplexe der Schafe und Ziegen. Zeit. Zellforsch. u. mikro. Anat., 13, 5I I-522, I93I.

TJIO (J. H.) and LEVAN (A.). - Comparative idiogram of the rat and the Yoshida rat sarcoma. Hereditas, 42, 218-234, 1956.

VEXGE (O.). - Variation in the spermatogonial chromosome number in rabbits. Kungl. Lantbrukshögskolans Annaler, 19, 233-24I, I952.

VEXGE (O.). - A simplified method for spreading the chromosomes in the rabbit blastocyst. Nature, 174, 608, I954.

Wahrman (J.) and Zahavi (A.). - Cytological contributions to the phylogeny and classification of the rodent genus Gerbillus. Nature, 175, 600-602, I955.

WODSEDAiEk (J. R.). - Studies on the cells of sheep with reference to spermatogenesis, oogenesis and sex determination. Anat. Rec., 23, I03, I922. 